\title{
Effect of pressurised intraperitoneal aerosol chemotherapy on the survival rate of patients with peritoneal carcinomatosis of gastric origin
}

\section{Fatah Tidadini}

CHU Grenoble: Centre Hospitalier Universitaire Grenoble Alpes

\section{Julio Abba}

CHU Grenoble: Centre Hospitalier Universitaire Grenoble Alpes

Jean-Louis Quesada

CHU Grenoble: Centre Hospitalier Universitaire Grenoble Alpes

\section{Magalie Baudrant}

CHU Grenoble: Centre Hospitalier Universitaire Grenoble Alpes

\section{Aline Bonne}

CHU Grenoble: Centre Hospitalier Universitaire Grenoble Alpes

\section{Alison Foote}

CHU Grenoble: Centre Hospitalier Universitaire Grenoble Alpes

Jean-Luc Faucheron

CHU Grenoble: Centre Hospitalier Universitaire Grenoble Alpes

\section{Olivier Glehen}

CHU Lyon: Hospices Civils de Lyon

\section{Laurent Villeneuve}

CHU Lyon: Hospices Civils de Lyon

Catherine Arvieux ( $\sim$ cArvieux@chu-grenoble.fr)

CHU Grenoble: Centre Hospitalier Universitaire Grenoble Alpes https://orcid.org/0000-0001-6674-799X

\section{Research Article}

Keywords: pressurised intraperitoneal aerosol chemotherapy (PIPAC), peritoneal carcinoma, gastric cancer, overall survival, systemic chemotherapy

Posted Date: August 3rd, 2021

DOl: https://doi.org/10.21203/rs.3.rs-754839/v1

License: (c) (1) This work is licensed under a Creative Commons Attribution 4.0 International License. Read Full License 


\section{Abstract}

INTRODUCTION: Pressurised intraperitoneal aerosol chemotherapy (PIPAC) is a new surgical technique, developed for the treatment of initially unresectable peritoneal carcinomatosis (PC). The objective of this study was to compare the results of PIPAC associated with systemic chemotherapy (PIPAC_CHEM) with those of systemic chemotherapy alone (ONLY_CHEM) in patients with gastric PC without metastasis other than peritoneal, and WHO performance status $<3$.

METHODS: This was a retrospective, single centre, comparative and non-randomised study. Seventeen PIPAC_CHEM patients were compared to 29 ONLY_CHEM patients. The primary endpoint was overall survival at 6 months from diagnosis of PC.

RESULTS: 98 patients were screened and 46 were included (PIPAC_CHEM, $n=17$; ONLY_CHEM, $n=29$ ). The 6-month survival rate was significantly higher in the PIPAC_CHEM group than in the ONLY_CHEM group 16/17 (94.1\% [65-99.2]) vs 19/29 (65.5\% [45.4-79.7]), respectively; $p=0.029$.

The median total hospital stay at 6 months was significantly shorter for PIPAC_CHEM (median 2 days [2-7]) vs (median 11 days [3-21]) $(p=0.045)$. Over the entire follow-up, the median survival $[95 \% \mathrm{Cl}]$ in PIPAC_CHEM was 12.8 months [7.2-34.3] vs 9.1 months [5.4-11.5] for the ONLY_CHEM group; $p=0.056$.

CONCLUSION: The overall survival at 6 months after the diagnosis of carcinomatosis was significantly better for PIPAC_CHEM patients. This difference appears to continue until at least 18 months. The total hospital stay at 6 months was significantly shorter in the PIPAC_CHEM group.

\section{Introduction}

Gastric cancer is the fifth most common cancer worldwide and the third leading cause of cancer deaths [1]. Peritoneal carcinomatosis (PC) is a very common manifestation in advanced adenocarcinoma-type gastric cancer [2]. Without intervention the prognosis is poor with survival of only a few months (median, 3.1 months) $[3,4]$. Standard treatment is based on systemic chemotherapy, however, the pharmacokinetics of drug delivery to the peritoneum is poor with limited efficacy compared to other metastatic sites such as the liver or lung [5]. When the carcinomas are resectable, selected patients can benefit from targeted therapeutic approaches combining peritonectomy and Hyperthermic Intraperitoneal Chemotherapy (HIPEC), offering a significant improvement in survival $[6,7]$.

For initially unresectable cases, in 2012 Reymond et al. described a new approach consisting of applying chemotherapy agents directly to the peritoneum using a pressurised aerosol, known as pressurised intraperitoneal aerosol chemotherapy (PIPAC) [8]. The use of this technique results in a higher local drug concentration compared to standard intraperitoneal or intravenous chemotherapy. The aim of this innovative therapy is to improve survival while preserving quality of life, but a further advantage is that it can render the PC resectable; more accessible to complete cytoreductive surgery (CRS). PIPAC has been applied to PC of all origins. Several recent studies have provided evidence that a treatment regimen 
combining PIPAC and systemic chemotherapy improved survival without adversely affecting quality of life [9-12]. However, in the absence of published prospective controlled trials, a clear advantage of this treatment regimen over systemic chemotherapy alone has not been established and the results in terms of efficacy and the benefit/risk ratio of this procedure remain debated. We conducted a retrospective study (PIPAC_GRE) comparing the results of PIPAC administered alternately with systemic chemotherapy (PIPAC_CHEM) versus systemic chemotherapy alone (ONLY-CHEM) in patients with non-resectable gastric $P C$ eligible for non-palliative treatment. The main objective was overall survival at 6 months from diagnosis of carcinomatosis. The secondary objectives were the duration of hospitalization up to 6 months after the diagnosis of carcinomatosis, overall survival throughout the study follow-up from the diagnosis of carcinomatosis and from the diagnosis of the primary tumour.

\section{Methods}

This was a retrospective, comparative and non-randomised study performed at Grenoble Alpes University Hospital, a centre with expertise in the PIPAC technique, between July 2016 and September 2020. The study population consisted of adult patients with adenocarcinoma-type gastric non-resectable PC, without metastasis other than peritoneal, treated either with PIPAC administered in alternation with systemic chemotherapy (PIPAC_CHEM) or by systemic chemotherapy alone (ONLY_CHEM). All patients receiving PIPAC in our hospital during the study period were included. The patients in the ONLY_CHEM control group had been treated during the same period and were carefully selected according to age, general state of health (WHO performance status $<3$ ) and absence of extra-peritoneal metastases so as to match as closely as possible the patients in the PIPAC group. Patient vital status of was determined from medical records and from phone calls, using September 18, 2020 as the point date. The times were calculated from the dates of diagnosis of carcinomatosis and of the primary tumour. The lengths of hospital stays during the first 6 months after the diagnosis of carcinomatosis were summed.

\section{The oncology strategy}

PIPAC procedures were performed every 6 to 8 weeks, alternating with systemic chemotherapy, replacing a cycle of intravenous chemotherapy. The treatment strategy and systemic chemotherapy used between PIPAC procedures and in the control group (ONLY_CHEM) had been decided by a multidisciplinary committee. Tumour response was based on surgical assessment using the Peritoneal Cancer Index ( $\mathrm{PCl})$ [13], routine intraoperative biopsies, and regular CT scans of the abdomen and chest.

\section{Surgical procedure and PIPAC}

The drug administration protocol for PIPAC was established in consultation with the specialized hospital pharmacist. All surgeons involved in PIPAC procedures had completed PIPAC training [14]. Procedures were performed in a dedicated operating room and a team of specialist operating room nurses assigned to PIPAC procedures. A safety checklist established before the first intervention jointly by the surgeons, the operating room nurses and the pharmacist was used at the 3 different stages of each procedure. 
PIPAC procedures were performed as previously described $[8,15]$. Briefly, under general anaesthesia, a laparoscopy was performed using an open laparoscopic technique to prevent intestinal wounds, which would contraindicate the procedure. A $12 \mathrm{mmHg}$ pneumoperitoneum was created, and to ensure the safety of the procedure the pressure of the pneumoperitoneum was maintained constant throughout the procedure. Two laparoscopic balloon trocars (of 11 and $12 \mathrm{~mm}$ ) were used. A thorough exploration of the abdomen, Peritoneal Cancer Index assessment, peritoneal biopsies, and ascites cytology (in the case of ascites) were performed prior to administration of the chemotherapy aerosol.

For carcinomas of gastric origin, Doxurubicin at a dose of $1.5 \mathrm{mg} / \mathrm{m}^{2}$ was administered in combination with Cisplatin $7.5 \mathrm{mg} / \mathrm{m}^{2}$ diluted in 40 and $150 \mathrm{~mL}$ of $0.9 \%$ sodium chloride, respectively. In the case of contraindication, Oxaliplatine at a dose of $92 \mathrm{mg} / \mathrm{m}^{2}$ in a $5 \%$ dextrose solution was recommended.

A CE certified CAPNOPEN® nebuliser (Reger Medizintechnik, GmbH, Villingendorf, Allemagne) connected to a high pressure injector was inserted through the trocars. Nebulisation of the drug was initiated by remote control after all staff had left the operating room. The nebulizer aerosolized the fluids at 30 $\mathrm{mL} / \mathrm{min}$ with a maximum pressure of 20 bars over 5 minutes. After 30 minutes of application, the $\mathrm{CO}_{2}$ and remaining toxic aerosols were evacuated by a closed air evacuation system. The safety checklist was systematically verified before administration of the drug, at the end of the procedure, and signed by the surgeon in charge and the operating room nurse.

\section{Follow-up}

Following each PIPAC procedure patients were kept under surveillance for at least 24 hours and a blood sample was taken on day 1 . Early mobilization and nutrition were standard for all patients. All signs of toxicity and/or postoperative complications occurring during the first 30 postoperative days were graded according to the Clavien-Dindo classification [16], with grades 3 to 5 corresponding to major complications. All patients (including the ONLY-CHEM control group) had a standard oncology follow-up with regular CT scans, tumour marker tests and clinical examinations.

\section{Statistical analysis}

Continuous data are presented using descriptive statistics median and [25th-75th percentiles]). Categorical data are presented using frequencies and percentages. Quantitative parameters were compared between groups using a Student's $t$ test, or a Mann-Whitney test when normality was rejected. Qualitative parameters were compared using the Chi-square test or otherwise a Fisher's exact test, as applicable.

Overall survival (OS) was defined as the time from the date of PC diagnosis until death due to any cause.

Survival rates were calculated using the Kaplan-Meier method. Overall survival at 6 months by treatment group was compared using a Log-Rank test. The role of confounding factors was explored using univariate Cox proportional risk models. 
A Cox proportional hazard multivariate model, with backward-stepwise selection (and a $20 \%$ threshold), was used to evaluate overall survival at 6 months for each treatment group.

A threshold of $5 \%$ was used to define the significance of the statistical tests. No adjustment for multiplicity was applied. Statistical analysis was performed using Stata software version 14.2 (STATA, StataCorp, Texas, USA).

\section{Results}

\section{Patients' characteristics}

Out of 98 consecutive patients presenting with gastric non-resectable PC between July 2016 and September 2020, Fifty-two (53.1\%) were excluded from the analysis (28 for extraperitoneal metastases, 9 too weak to be treated, 9 for missing dates of diagnosis and / or death and 6 for gastrointestinal stromal tumours). Forty-six (46.9\%) treated patients were included on the study; seventeen had received PIPAC (PIPAC_CHEM) and 29 matched controls had received systemic chemotherapy only (CHEM_ONLY) (Fig. 1).

The demographic data of all individuals included in the study, the characteristics of the tumours and carcinomatosis, and any previous surgery are presented on Table 1. Twenty-eight patients were male (60.9\%). At the time of diagnosis of carcinomatosis: the median age was 68.5 [57-74], and median BMI was 22.0 [19.1-26]. Twenty-four patients (52.2\%) had presented an undifferentiated adenocarcinoma and 27 PC (58.7\%) were classed as synchronous. All patients had received at least one line of intravenous chemotherapy (Table 2). The patients in the two groups (PIPAC_CHEM and ONLY_CHEM) were comparable for all characteristics except age, with the PIPAC_CHEM group being significantly younger (median 64 years [56-68] vs 73.5 years [61-79]; $p=0.005$ ). The 17 PIPAC_CHEM patients underwent a total of 42 PIPAC procedures, with a median of 2 procedures $[1 ; 3]$ and median $\mathrm{PCl}$ at the start of treatment of 18 [12-20]. 
Table 1

- Demographic and medical characteristics

\begin{tabular}{|c|c|c|c|c|}
\hline & $\begin{array}{l}\text { Population } \\
n=46\end{array}$ & $\begin{array}{l}\text { PIPAC_CHEM } \\
\mathrm{n}=17\end{array}$ & $\begin{array}{l}\text { ONLY_CHEM } \\
n=29\end{array}$ & p-value \\
\hline Age & $\begin{array}{l}68.5[57- \\
74]\end{array}$ & $64[56-68]$ & 74 [61 -79] & $0.005^{(4)}$ \\
\hline Sex (Male) & $60.9 \%(28)$ & $58.8 \%(10)$ & $62.1 \%(18)$ & $0.828^{(1)}$ \\
\hline BMI & $\begin{array}{l}22[19.1- \\
26]\end{array}$ & $\begin{array}{l}21.3[18.6- \\
24.7]\end{array}$ & $\begin{array}{l}22.2[20.4- \\
27.4]\end{array}$ & $0.407^{(3)}$ \\
\hline WHO Performance Status & $15.6 \%(7)$ & $29.4 \%(5)$ & $7.1 \%(2)$ & $0.111^{(2)}$ \\
\hline 0 & $55.6 \%(25)$ & $41.2 \%(7)$ & $64.3 \%(18)$ & \\
\hline 1 & $28.9 \%(13)$ & $29.4 \%(5)$ & $28.6 \%(8)$ & \\
\hline \multicolumn{5}{|l|}{2} \\
\hline \multicolumn{5}{|l|}{ Medical history } \\
\hline COPD / Resp. patho & $28.3 \%(13)$ & $23.5 \%(4)$ & $31 \%(9)$ & $0.739^{(2)}$ \\
\hline Dyslipidemia & $15.2 \%(7)$ & $11.8 \%(2)$ & $17.2 \%(5)$ & $1.000^{(2)}$ \\
\hline Hypertension & $32.6 \%(15)$ & $29.4 \%(5)$ & $34.5 \%(10)$ & $0.723^{(1)}$ \\
\hline Cardiopathy & $10.9 \%(5)$ & $5.9 \%(1)$ & $13.8 \%(4)$ & $0.640^{(2)}$ \\
\hline Stroke & $2.2 \%(1)$ & $5.9 \%(1)$ & $0 \%(0)$ & $0.370^{(2)}$ \\
\hline Atrial fibrillation & $8.7 \%(4)$ & $11.8 \%(2)$ & $6.9 \%(2)$ & $0.619^{(2)}$ \\
\hline Peripheral artery disease & $0 \%(0)$ & $0 \%(0)$ & $0 \%(0)$ & - \\
\hline $\begin{array}{l}\text { Deep vein thrombosis / pulmonary } \\
\text { embolism }\end{array}$ & $4.4 \%(2)$ & $5.9 \%(1)$ & $3.5 \%(1)$ & $1.000^{(2)}$ \\
\hline Diabetes & $13 \%(6)$ & $5.9 \%(1)$ & $17.2 \%(5)$ & $0.390^{(2)}$ \\
\hline Renal failure & $8.7 \%(4)$ & $5.9 \%(1)$ & $10.3 \%(3)$ & $1.000^{(2)}$ \\
\hline Hepathopathy & $4.4 \%(2)$ & $5.9 \%(1)$ & $3.5 \%(1)$ & $1.000^{(2)}$ \\
\hline Abdominal Surgery & $28.3 \%(13)$ & $23.5 \%(4)$ & $31 \%(9)$ & $0.739^{(2)}$ \\
\hline
\end{tabular}

Median with [25th - 75th percentiles], frequency(Number)

Chi-2 test ${ }^{(1)}$, Fisher-exact test ${ }^{(2)}$, Student t-test ${ }^{(3)}$, Mann-Whitney test ${ }^{(4)}$ 


\begin{tabular}{|c|c|c|c|c|}
\hline & $\begin{array}{l}\text { Population } \\
n=46\end{array}$ & $\begin{array}{l}\text { PIPAC_CHEM } \\
\mathrm{n}=17\end{array}$ & $\begin{array}{l}\text { ONLY_CHEM } \\
n=29\end{array}$ & p-value \\
\hline Other cancer & $24.4 \%(11)$ & $17.7 \%(3)$ & $28.6 \%(8)$ & $0.493^{(2)}$ \\
\hline Other medical history & $73.9 \%(34)$ & $88.2 \%(15)$ & $65.5 \%(19)$ & $0.163^{(2)}$ \\
\hline Gastrectomy before carcinomatosis & $21.7 \%(10)$ & $11.8 \%(2)$ & $27.6 \%(8)$ & $0.282^{(2)}$ \\
\hline Partial & $30 \%(3)$ & $0 \%(0)$ & $37.5 \%(3)$ & $1.000^{(2)}$ \\
\hline Total & $70 \%(7)$ & $100 \%(2)$ & $62.5 \%(5)$ & \\
\hline Peritoneal carcinomatosis & $58.7 \%(27)$ & $70.6 \%(12)$ & $51.7 \%(15)$ & $0.210^{(1)}$ \\
\hline Synchrone & $41.3 \%(19)$ & $29.4 \%(5)$ & $48.3 \%(14)$ & \\
\hline \multicolumn{5}{|l|}{ Metachrone } \\
\hline Differentiated adenocarcinoma & $47.8 \%(22)$ & $47.1 \%(8)$ & $48.3 \%(14)$ & $0.936^{(1)}$ \\
\hline \multirow[t]{2}{*}{$\begin{array}{l}\text { Time between metachrone carcinomatosis } \\
\text { and primitive tumour diagnosis (Days) }\end{array}$} & $\begin{array}{l}270[142- \\
719]\end{array}$ & $\begin{array}{l}362[230- \\
1845]\end{array}$ & $\begin{array}{l}240.5 \\
{[142-661]}\end{array}$ & $0.517^{(4)}$ \\
\hline & $\mathrm{n}=19$ & $n=5$ & $\mathrm{n}=14$ & \\
\hline $\begin{array}{l}\text { Number of hospital stays } 6 \text { months after } \\
\text { diagnosis of carcinomatosis }\end{array}$ & $1[1-2]$ & $1[1-1]$ & $1[1-2]$ & $\begin{array}{l}0.288 \\
(4)\end{array}$ \\
\hline Number of PIPAC procedures & $2[1-3]$ & $2[1-3]$ & - & - \\
\hline Initial PCI & 18 [12-20] & 18 [12-20] & - & - \\
\hline \multicolumn{5}{|c|}{ Median with [25th - 75th percentiles], frequency(Number) } \\
\hline 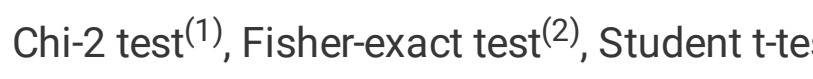 & 3), Mann-W & ey test ${ }^{(4)}$ & & \\
\hline
\end{tabular}


Table 2

- Systemic Chemotherapy

\begin{tabular}{|lllll|}
\hline Chemotherapy & Whole study Population $\mathbf{n = 4 6}$ & $\begin{array}{l}\text { PIPAC_CHEM } \\
\mathbf{n}=17\end{array}$ & $\begin{array}{l}\text { ONLY_CHEM } \\
\mathbf{n}=\mathbf{2 9}\end{array}$ & P-value \\
\hline FOLFOX & $65.2 \%(30)$ & $82.4 \%(14)$ & $55.2 \%(16)$ & $0.062^{(1)}$ \\
\hline FOLFIRI & $4.4 \%(2)$ & $0 \%(0)$ & $6.9 \%(2)$ & $0.524^{(2)}$ \\
\hline LV5FU2 & $10.9 \%(5)$ & $17.7 \%(3)$ & $6.9 \%(2)$ & $0.343^{(2)}$ \\
\hline FU & $8.7 \%(4)$ & $0 \%(0)$ & $13.8 \%(4)$ & $0.281^{(2)}$ \\
\hline TAXOL & $15.2 \%(7)$ & $5.9 \%(1)$ & $20.7 \%(6)$ & $0.234^{(2)}$ \\
\hline RAMUCIRUMAB & $4.4 \%(2)$ & $5.9 \%(1)$ & $3.5 \%(1)$ & $1.000^{(2)}$ \\
\hline OXALIPLATINE & $10.9 \%(5)$ & $5.9 \%(1)$ & $13.8 \%(4)$ & $0.637^{(2)}$ \\
\hline FLUORURACILE & $2.2 \%(1)$ & $5.9 \%(1)$ & $0 \%(0)$ & $0.370^{(2)}$ \\
\hline CISPLATINE & $4.4 \%(2)$ & $5.9 \%(1)$ & $3.5 \%(1)$ & $1.000^{(2)}$ \\
\hline TAXOTERE & $2.2 \%(1)$ & $5.9 \%(1)$ & $0 \%(0)$ & $0.370^{(2)}$ \\
\hline HERCEPTIN & $2.2 \%(1)$ & $0 \%(0)$ & $3.5 \%(1)$ & $1.000^{(2)}$ \\
\hline CARBOPLATINE & $4.4 \%(2)$ & $0 \%(0)$ & $6.9 \%(2)$ & $0.524^{(2)}$ \\
\hline DOCETAXEL & $4.4 \%(2)$ & $0 \%(0)$ & $6.9 \%(2)$ & $0.524^{(2)}$ \\
\hline Frequency(Number), Chi-2 test ${ }^{(1)}$, Fisher-exact test $(2)$ & & \\
\hline & & & & \\
\hline
\end{tabular}

Overall survival at 6 months from diagnosis of carcinomatosis

The 6-month survival rate [95\% Cl] was significantly better for PIPAC_CHEM vs ONLY_CHEM groups: 16/17 (94.1\% [65-99.2]) vs 19/29 (65.5\% [45.4-79.7]), respectively; $p=0.029$. (Fig. 2).

Duration of hospital stay 6 months after diagnosis of carcinomatosis

The median total length of hospital stay at 6 months was 7 days [2-17]. As shown in Fig. 3, patients in the PIPAC_CHEM had a shorter total length of hospitalisation at 6 months compared to the ONLY_CHEM (median 2 [2-7] days vs 11 [3-21]; $p=0.045$ ).

Overall survival after the diagnosis of carcinomatosis 
The median survival [95\% Cl] for the whole study population was 10.2 months [7.3-12]. The median survival [95\% Cl] in the PIPAC_CHEM group was 12.8 months [7.2-34.3] versus 9.1 months [5.4-11.5] for the ONLY_CHEM group $(p=0.056)$, see supplementary eTable 1 . At 12 and 18 months OS rates [95\% $\mathrm{Cl}]$ were, $51.8 \%$ [26.2-72.4] and 38.8\% [16.3-61.1] respectively for the PIPAC_CHEM group versus 31.8\% [15.9-48.9] and 15.9\% [5.2-32] respectively, for the ONLY_CHEM group (Fig. 4).

\section{Overall survival after primary tumour diagnosis.}

The rate of OS at 6 months after primary tumour diagnosis was not significantly different between PIPAC_CHEM and ONLY_CHEM (16/17 (94.1\%) vs 25/29 (86.2\%); $\mathrm{p}=0.391)$, eFigure 1 in supplement. Over the entire follow-up, the median survival [95\% CI] in the PIPAC_CHEM group was 31 months [9.8$75.5]$ vs. 13.6 [11-23.5]; with OS rates [ $95 \% \mathrm{Cl}$ ] at 12,18 and 24 months of $64.2 \%$ [36.9-82.1], $57.8 \%$ [31.1-77.3] and $51.3 \%$ [25.7-72.1] vs $62.1 \%$ [42.1-76.9], 36.5\% [19.5-53.8] and $21.9 \%$ [9-38.5] respectively; see eFigure 2 in supplement.

\section{Radical surgery after treatment}

The rate of radical surgery in the PIPAC_CHEM group was $2 / 17$ (11.8\%) vs $3 / 29(10.3 \%)$ for the ONLY_CHEM group; (non-significant difference $p=1.000$; eTable 2 in supplement).

Major complications classified Clavien 3b or 4, over the entire follow-up (from diagnosis of carcinomatosis)

The rate of major complications classified Clavien 3b or 4 in the PIPAC_CHEM group was 2/17 (11.8\%) vs $2 / 29(6.9 \%)$ for the ONLY_CHEM group; (non-significant difference $p=0.619$; eTable 3 in supplement).

\section{Multivariate analysis}

We looked for confounding factors on which it would be interesting to adjust the comparison of 6-month survival between groups. The significance threshold for the selection of these parameters was set at $20 \%$. Univariate analyses using a Cox model were performed on the following risk factors: age, sex, BMI, WHO performance status, previous gastrectomy, histology of the primary tumour, chronology of onset of carcinomatosis, and time to diagnosis of cancer (eTable 4). The analyses were not performed on $\mathrm{PCl}$ risk factor because ONLY-CHEM patients did not have an exploratory laparoscopy ( $\mathrm{PCl}$ score not available for patients in this group). None of these parameters, in particular age, emerged as a risk factor. Only the WHO performance status was retained as a confounding factor, probably linked to death at 6 months (HR $[95 \% \mathrm{Cl}], 1.93[0.75 ; 4.98])$, see eTable 5 in supplement. The comparison of overall survival at 6 months between the chemotherapy groups before $(p=0.062)$ or after adjustment for the WHO performance status $(p=0.062)$ using the multivariate Cox model suggests a protective trend of PIPAC treatment associated with systemic chemotherapy compared to systemic chemotherapy alone (HR [95\% Cl], 0.141 [0.018; 1.104]) but was not statistically significant; eTable 5 in supplement. 


\section{Discussion}

In this non-randomised retrospective study, we compared overall survival following two strategies of treatment for selected patients with adenocarcinoma-type gastric cancer and unresectable peritoneal carcinomatosis. Treatment with PIPAC given alternately with systemic chemotherapy provided a significant difference $(p=0.029)$ in OS at 6 months $[95 \% \mathrm{Cl}]$ after diagnosis of PC compared with systemic chemotherapy alone (94.1\% [65-99.2] and 65.5\% [45.4-79.7] respectively). The total hospital stay at 6 months was significantly shorter in the PIPAC_CHEM group $(p=0.045)$.

After a median follow-up of 10.2 months $(0.4,43.6)$ for the entire study population, the median survival was longer in the PIPAC_CHEM group (difference 3.7 months). PIPAC appears to delay oncologic progression with a significant effect on 6-month survival that appears to continue for at least 18 months. The characteristics of the carcinomatosis, of the tumour, and any previous gastrectomy did not appear to be significantly different between the groups, however these characteristics seemed more favourable in the ONLY_CHEM group. PIPAC treatment appears all the more promising in the medium term. In a metaanalysis of 106 articles or case reports of PIPAC, including 45 clinical studies, Alyami et al.[12] reported OS to be between 8 and 15 months. In their recent study the same author reports survival of 19.1 months [11]. Thus, our results (median survival [ $95 \% \mathrm{Cl}$ ] of 12.8 months [7.2-34.3]) appear to be in line with most of the published studies. However, whatever the treatment strategy, the long-term prognosis remains poor.

The median survival $[95 \% \mathrm{Cl}$ ] from diagnosis of PC, with chemotherapy alone seen in our study $(9.1$ months [5.4-11.5]) is in the same range as that reported in the literature. Thomassen et al. estimated survival [95\% Cl] to be 8.0 months [6.8-9.3], and 9.9 months [7.7-11.8] for patients having undergone primary tumour resection [17]. A direct comparison is difficult due to the heterogeneous treatments in our ONLY_CHEM group, chemotherapy and resection of the primary tumour (27.6\% (8)); and also the evolution over time of chemotherapy protocols for this indication. Nevertheless, median survival of ONLY_CHEM patients was similar to those reported in the literature.

Over the entire follow-up from diagnosis of carcinomatosis, two major complications classified Clavien $3 \mathrm{~b}$ and 4 occurred, in the PIPAC_CHEM group, a case of obstructive jaundice which was operated by laparotomy and a hemorrhagic shock requiring a passage in intensive care; and in the ONLY_CHEM group, one patient had an exploratory laparotomy for cardiac arrest due to a coronary spasm and another exploratory laparotomy for occlusion. see eTable 3 in supplement.

Among the patients who are still alive, two patients in the PIPAC_CHEM group have had total gastrectomy associated with CRS and HIPEC; and in the ONLY_CHEM group, one patient has undergone partial gastrectomy associated with CRS and HIPEC and another has had CRS without HIPEC (due to his age and comorbidities). A third patient in this group died following a total gastrectomy associated with CRS and HIPEC; see eTable 2 in supplement.

The comparison of major complications classified as Clavien $3 \mathrm{~b}$ or $4(\mathrm{P}=0.619)$ and the number of hospital stays $(p=0.288)$, respectively over the entire follow-up and at 6 months after the diagnosis of 
carcinomatosis did not show any significant difference between the 2 groups. Even so, the duration of hospital stay at 6 months, was significantly shorter in the PIPAC_CHEM group $(p=0.045)$. PIPAC treatment did not affect the quality of life of patients.

Finally, our study has some limitations: first, it was a single-center retrospective study with a relatively small sample size. Second, was the absence of randomisation. Two prospective randomized studies (PIPAC associated with systemic chemotherapy vs systemic chemotherapy alone) are currently ongoing in France (PIPAC EstoK 01- NCT04065139) [18] and in Germany (EudraCT 2018-001035-40) [19] to evaluate the benefit of PIPAC in patients with gastric PC. The results of these studies will provide more information on the effect of PIPAC on overall survival in this indication.

\section{Conclusion}

In this retrospective study, overall survival at 6 months after the diagnosis of carcinomatosis was significantly better for patients who received PIPAC than for similar patients treated with chemotherapy alone; and this difference appears to persist until at least 18 months. The significantly shorter total length of hospitalisation for the PIPAC group suggests that this strategy may be the most appropriate for the treatment of gastric PC. These encouraging results justify further evaluation by prospective randomized trials that include quality of life and medico-economic analyses.

\section{Declarations}

\section{Authors' contributions:}

Study concept and design: Tidadini, Glehen, Villeneuve, Arvieux. Data acquisition: Tidadini, Abba, Arvieux. Quality control of data and algorithms: Tidadini, Abba, Bonne, Baudrant, Arvieux. Data analysis and interpretation: Tidadini, Abba, Quesada, Faucheron, Arvieux. Statistical analysis: Quesada. Manuscript preparation: Tidadini, Quesada, Foote, Arvieux. Manuscript editing: Tidadini, Foote, Bonne, Arvieux. Manuscript review: All Authors.

Funding: No funding was received for conducting this Study.

Conflicts of interest/Competing interests: None of the authors have a conflict of interest to declare.

Availability of data and material (data transparency): Anonymized patient data will be made available on reasonable request for academic use after publication of the article and with a signed contract between the applicant and Grenoble Alpes University Hospital.

Informed consent: Patients were informed that their anonymized data might in the future be the subject of a research protocol and could oppose this by informing the doctor.

Ethics approval: Study ethical approval was obtained on 03.02.2021 (CECIC Rhône-Alpes-Auvergne, Clermont-Ferrand, IRB 5891) and was registered in the CHU Grenoble Alpes register of studies respecting 
the reference methodology MR004 of the National Commission for Informatics and Liberties (CNIL).

Trial registration - clinicaltrials.gov Identifier: NCT 04879953

\section{References}

[1] Bray F, Ferlay J, Soerjomataram I, Siegel RL, Torre LA, Jemal A. Global cancer statistics 2018: GLOBOCAN estimates of incidence and mortality worldwide for 36 cancers in 185 countries. CA Cancer J Clin 2018; 68:394-424.

[2] Coccolini F, Cotte E, Glehen O, Lotti M, Poiasina E, Catena F, et al. Intraperitoneal chemotherapy in advanced gastric cancer. Meta-analysis of randomized trials. Eur J Surg Oncol 2014; 40:12-26.

[3] Sadeghi B, Arvieux C, Glehen O, Beaujard AC, Rivoire M, Baulieux J, et al. Peritoneal carcinomatosis from non-gynecologic malignancies: results of the EVOCAPE 1 multicentric prospective study. Cancer 2000; 88:358-63.

[4] Dehal A, Smith JJ, Nash GM. Cytoreductive surgery and intraperitoneal chemotherapy: an evidencebased review-past, present and future. J Gastrointest Oncol 2016; 7:143-57.

[5] Franko J, Shi Q, Meyers JP, Maughan TS, Adams RA, Seymour MT, et al. Prognosis of patients with peritoneal metastatic colorectal cancer given systemic therapy: an analysis of individual patient data from prospective randomised trials from the Analysis and Research in Cancers of the Digestive System (ARCAD) database. Lancet Oncol 2016; 17:1709-19.

[6] Bonnot P-E, Piessen G, Kepenekian V, Decullier E, Pocard M, Meunier B, et al. Cytoreductive Surgery With or Without Hyperthermic Intraperitoneal Chemotherapy for Gastric Cancer With Peritoneal Metastases (CYTO-CHIP study): A Propensity Score Analysis. J Clin Oncol 2019; 37:2028-40.

[7] van Driel WJ, Koole SN, Sikorska K, Schagen van Leeuwen JH, Schreuder HWR, Hermans RHM, et al. Hyperthermic Intraperitoneal Chemotherapy in Ovarian Cancer. N Engl J Med 2018; 378:230-40.

[8] Solaß W, Hetzel A, Nadiradze G, Sagynaliev E, Reymond MA. Description of a novel approach for intraperitoneal drug delivery and the related device. Surg Endosc 2012; 26:1849-55.

[9] Khomyakov V, Ryabov A, Ivanov A, Bolotina L, Utkina A, Volchenko N, et al. Bidirectional chemotherapy in gastric cancer with peritoneal metastasis combining intravenous XELOX with intraperitoneal chemotherapy with low-dose cisplatin and Doxorubicin administered as a pressurized aerosol: an open-label, Phase-2 study (PIPAC-GA2). Pleura Peritoneum 2016; 1:159-66.

[10] Nadiradze G, Giger-Pabst U, Zieren J, Strumberg D, Solass W, Reymond M-A. Pressurized Intraperitoneal Aerosol Chemotherapy (PIPAC) with Low-Dose Cisplatin and Doxorubicin in Gastric Peritoneal Metastasis. J Gastrointest Surg $2016 ; 20: 367-73$. 
[11] Alyami M, Bonnot P-E, Mercier F, Laplace N, Villeneuve L, Passot G, et al. Pressurized intraperitoneal aerosol chemotherapy (PIPAC) for unresectable peritoneal metastasis from gastric cancer. Eur J Surg Oncol $2021 ; 47: 123-7$.

[12] Alyami M, Hübner M, Grass F, Bakrin N, Villeneuve L, Laplace N, et al. Pressurised intraperitoneal aerosol chemotherapy: rationale, evidence, and potential indications. Lancet Oncol 2019;20: e368-77.

[13] Jacquet $\mathrm{P}$, Sugarbaker $\mathrm{PH}$. Clinical research methodologies in diagnosis and staging of patients with peritoneal carcinomatosis. Cancer Treat Res 1996; 82:359-74.

[14] Alyami M, Sgarbura O, Khomyakov V, Horvath P, Vizzielli G, So J, et al. Standardizing training for Pressurized Intraperitoneal Aerosol Chemotherapy. Eur J Surg Oncol 2020; 46:2270-5.

[15] Solass W, Kerb R, Mürdter T, Giger-Pabst U, Strumberg D, Tempfer C, et al. Intraperitoneal chemotherapy of peritoneal carcinomatosis using pressurized aerosol as an alternative to liquid solution: first evidence for efficacy. Ann Surg Oncol 2014; 21:553-9.

[16] Dindo D, Demartines N, Clavien P-A. Classification of surgical complications: a new proposal with evaluation in a cohort of 6336 patients and results of a survey. Ann Surg 2004; 240:205-13.

[17] Thomassen I, van Gestel YR, van Ramshorst B, Luyer MD, Bosscha K, Nienhuijs SW, et al. Peritoneal carcinomatosis of gastric origin: a population-based study on incidence, survival and risk factors. Int $\mathrm{J}$ Cancer 2014; 134:622-8.

[18] Eveno C, Jouvin I, Pocard M. PIPAC EstoK 01: Pressurized IntraPeritoneal Aerosol Chemotherapy with cisplatin and doxorubicin (PIPAC C/D) in gastric peritoneal metastasis: a randomized and multicenter phase II study. Pleura Peritoneum 2018; 3:20180116.

[19] Oliver Goetze T, Al-Batran S-E, Pabst U, Reymond M, Tempfer C, Bechstein WO, et al. Pressurized intraperitoneal aerosol chemotherapy (PIPAC) in combination with standard of care chemotherapy in primarily untreated chemo naïve upper gi-adenocarcinomas with peritoneal seeding - a phase II/III trial of the AIO/CAOGI/ACO. Pleura Peritoneum $2018 ; 3: 20180113$.

\section{Figures}




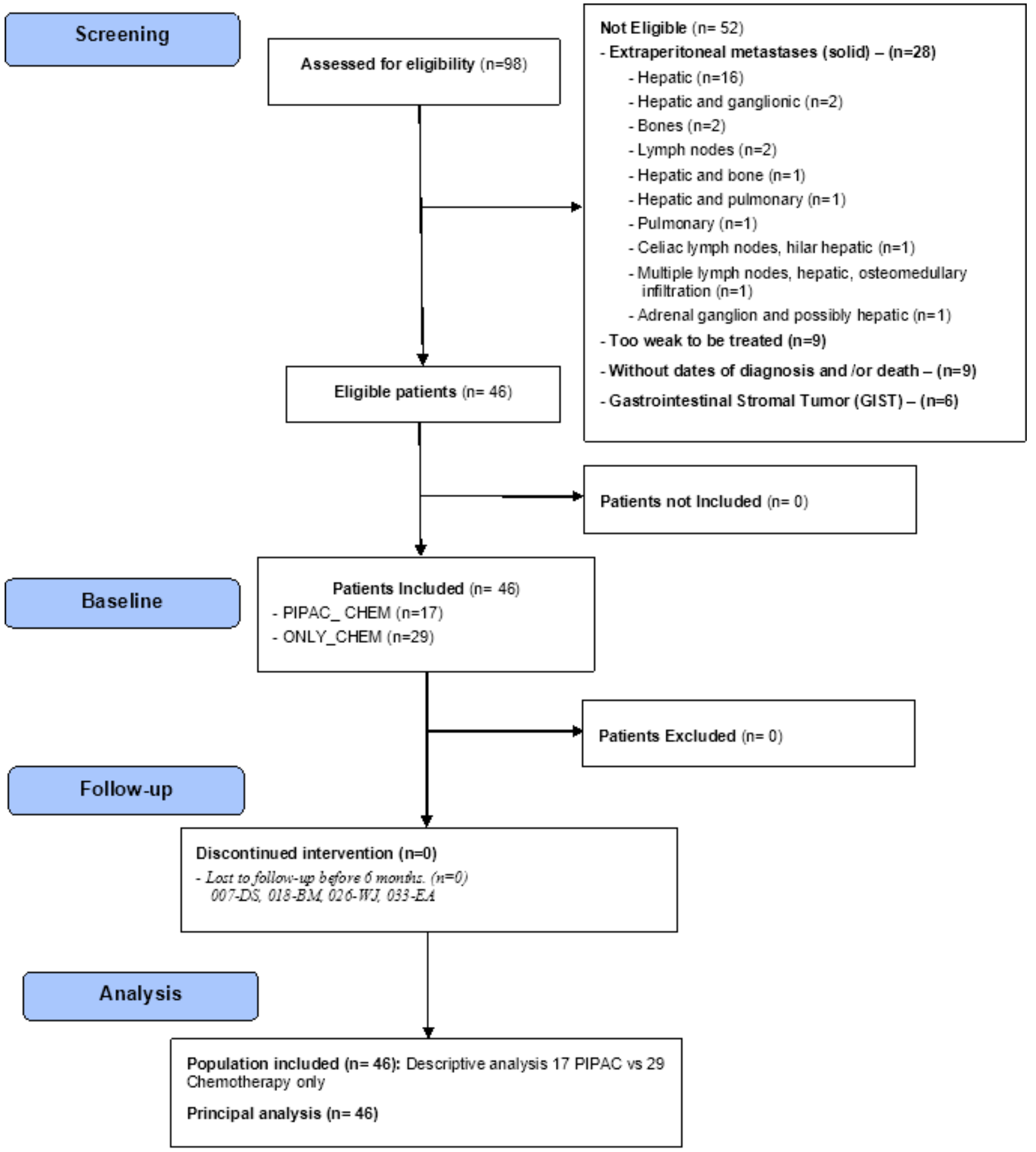

Figure 1

Flow diagram 


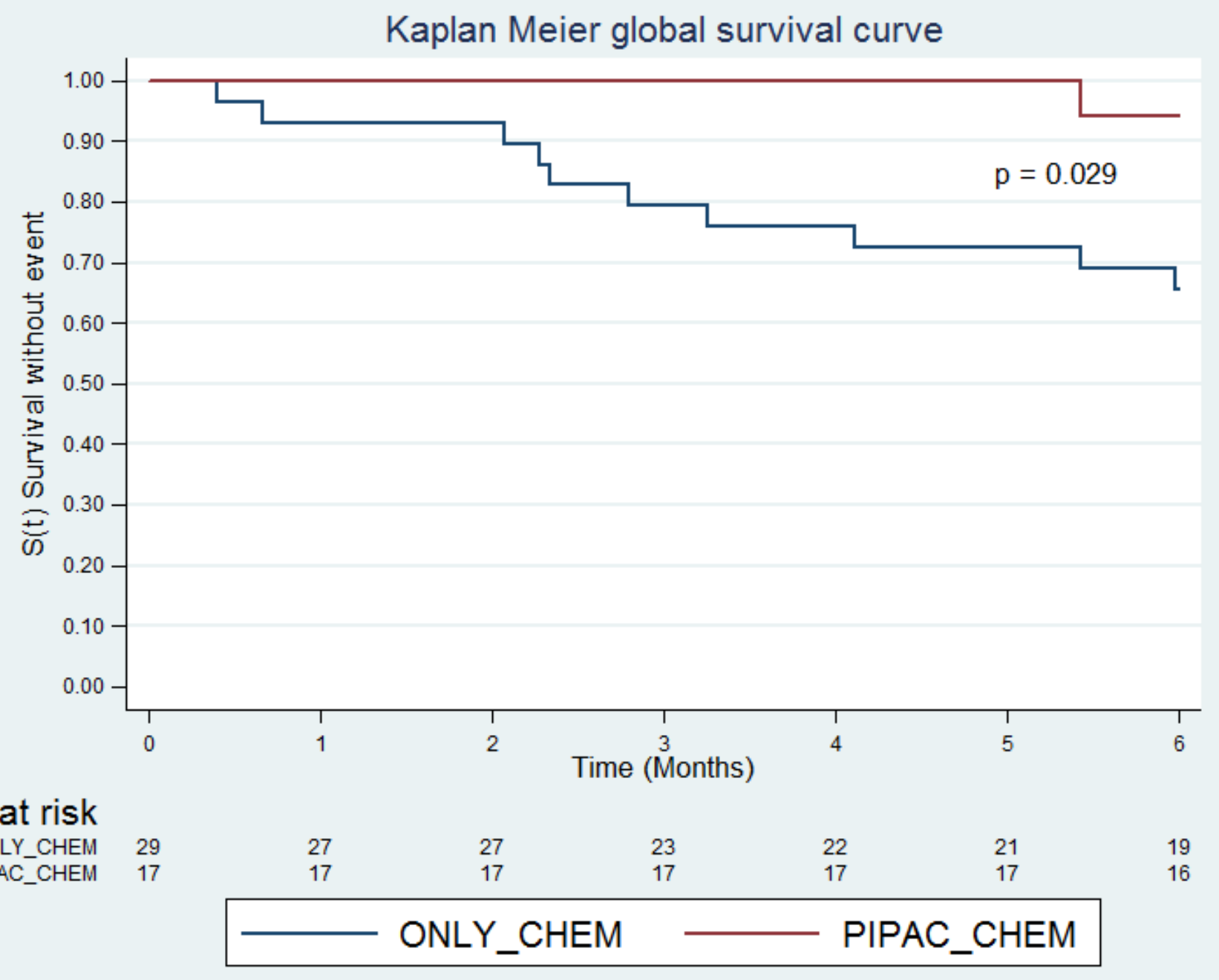

Figure 2

Kaplan Meier overall survival at 6 months follow-up (from diagnosis of carcinomatosis) 


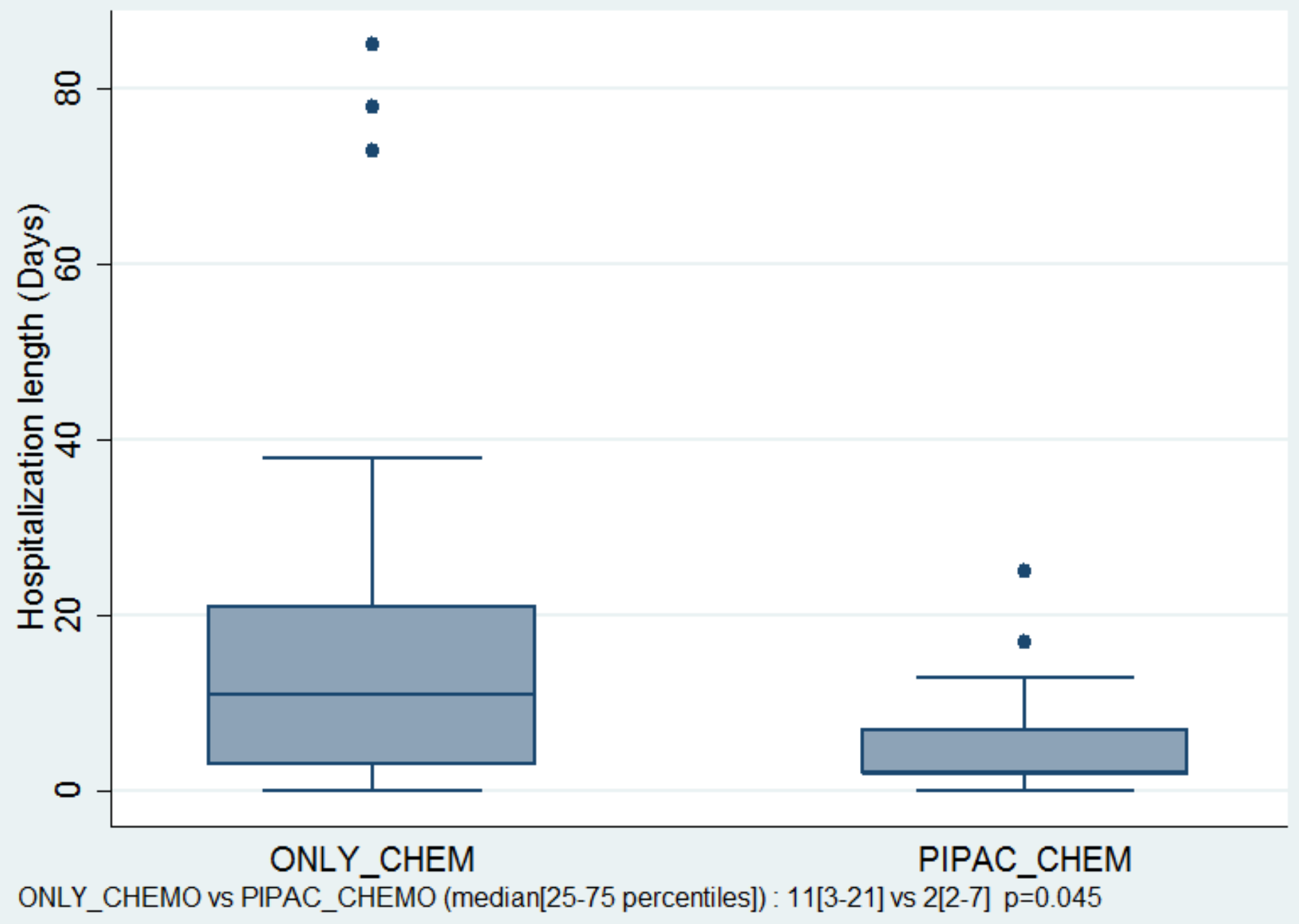

Figure 3

Comparison of the Total Length of hospital stay at 6 months follow-up (from diagnosis of carcinomatosis) 


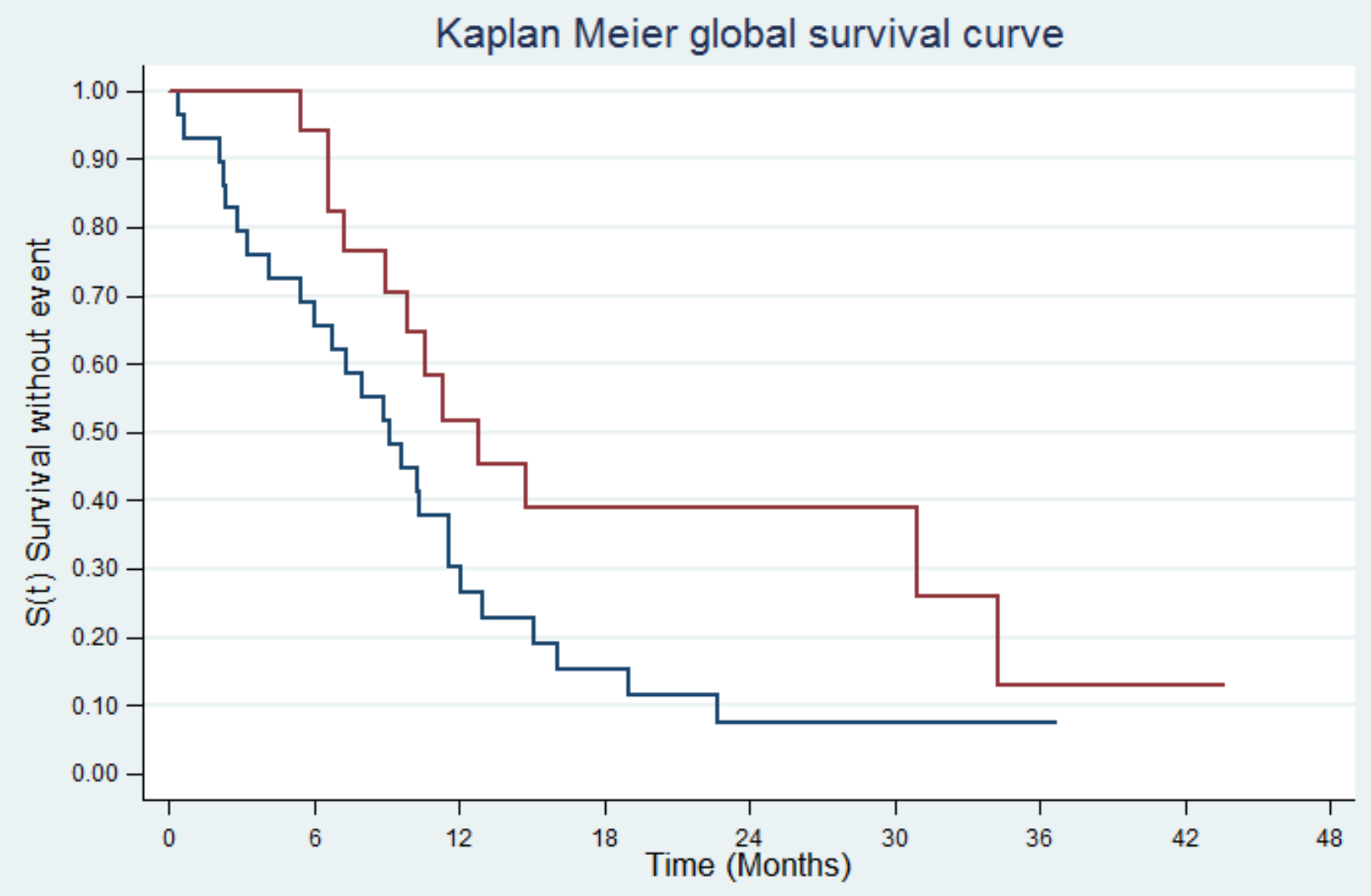

Number at risk

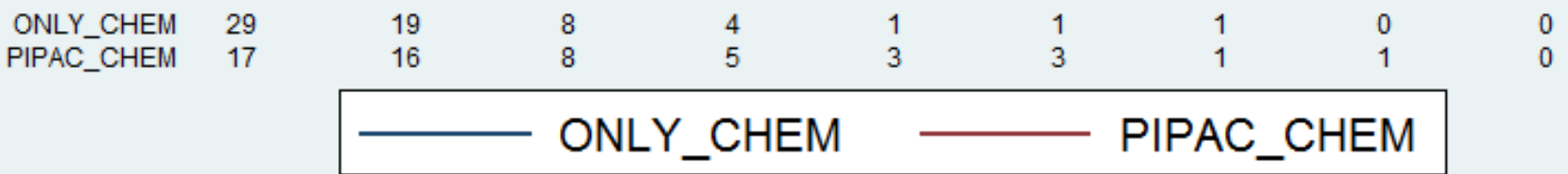

Figure 4

Kaplan Meier overall survival over the entire follow-up (from diagnosis of carcinomatosis)

\section{Supplementary Files}

This is a list of supplementary files associated with this preprint. Click to download.

- SupplementMateriel.docx 